\title{
Automatic Acquisition of Failure Mode and Effect Analysis Ontology for Sustainable Risk Management
}

\author{
Zobia Rehman ${ }^{1,2, *}$ (1), Claudiu Vasile Kifor ${ }^{2}$, Farhana Jabeen ${ }^{1}$, Sheneela Naz $^{1}$ and \\ Muhammad Waqar ${ }^{1}$ \\ 1 Department of Computer Science, COMSATS University Islamabad, Islamabad 44000, Pakistan; \\ farhanakhan@comsats.edu.pk (F.J.); sheneela.naz@comsats.edu.pk (S.N.); mwaqar@comsats.edu.pk (M.W.) \\ 2 Faculty of Engineering, Lucian Blaga University of Sibiu, 550024 Sibiu, Romania; claudiu.kifor@ulbsibiu.ro \\ * Correspondence: zobia.rehman@hotmail.com
}

Received: 28 September 2020; Accepted: 24 November 2020; Published: 7 December 2020

\begin{abstract}
In this piece of research, we have presented an approach to populate Failure Mode and Effect Analysis (FMEA) ontology from existing worksheets prepared by experts. FMEA is a commonly used method for risk assessment in any organization. This method is initiated by domain experts who analyze all the associated risks to a product or process, their causes, severity, effects and mitigation actions. Besides domain experts, time and cost are the other two factors involved in successful completion of FMEA. Reusability of the knowledge produced at the end of this method can bring numerous benefits to an organization. Some ontologies are available for semantic content management of FMEA knowledge but in order to avail their full benefits, it is must that they can acquire the existing knowledge automatically. Major objective of this article is to develop an algorithm, which can populate FMEA ontology from existing worksheets. Major contribution of this work is to identify an existing FMEA ontology and its evaluation for schema and relationship richness, then its automatic population using proposed algorithm without human intervention, and finally making it a part of complete knowledge management system. Our proposed algorithm correctly mapped 1357 instances to FMEA ontology from manually prepared FMEA spreadsheets. This FMEA ontology has been queried by domain experts and it was proved to be very helpful in experts like decision-making.
\end{abstract}

Keywords: knowledge management; risk management; FMEA; ontology acquisition

\section{Introduction}

Organizations these days are competing to develop sustainability strategies for seeking an increase in profits and reduction in risk. Enterprises have to deal timely with the rapid advancements in technology, changes in value chain methodology, and spontaneous unavailability of experts. This is why enterprises are seeking to establish robust risk management systems. Risk management is referred to the process of identification of potential risk in advance, its analysis and deciding certain precautions to mitigate it [1] Effective risk management helps organizations achieve their objectives and sustain in the market by developing a certain level of trust between organization, its stakeholders and customers.

Sustainability requires organizational knowledge base for problem-solving, which is usually built after extensive trial and error learning. It is also emphasized to facilitate implementation of Knowledge Management (KM) strategies in order to promote sustainability [2].

Similarly, Enterprise Risk Management (ERM) keeps company-wide perspective at the top during identification and analysis of risk. It develops a value bond between firms and shareholders. It is a structured approach to identify all the uncertain circumstances faced by an enterprise by aligning people, processes, and knowledge. It identifies and prioritizes all possible risks associated to a firm for better risk management and in time decision-making [3]. 
Failure Mode and Effect Analysis (FMEA) is a structured approach for discovering the risks associated to a product or process design. Sustainability of FMEA helps manufacturers in prioritizing failures, which need mitigation actions and require cause detection in order to improve product quality continuously [4]. This approach requires efforts and availability of experts. After the completion of this analysis, valuable knowledge is produced. Organizations keep this knowledge in spread sheets, which cannot be utilized further. Semantic content management of this knowledge is important for sustainable risk management.

In experts view, "Enterprise Content Management is a collection of strategies, processes, methods, systems and technologies, which are required for capturing, creating, managing, using, publishing, storing, preserving, and disposing contents within and between organizations" [5]. We believe that effective content management cannot be achieved until and unless an organization provides a centralized and semantic approach for preserving organizational contents. Contents related to each process that are carried out in an organization must be stored in structured documents, which are not only understandable for all users but also can be inferred for decision-making automatically.

Ontologies are used to represent domain knowledge formally. Ontologies are being largely used in knowledge management systems because they provide a structured representation of domain knowledge, enhance the understanding of the knowledge, and can be integrated in any information system. Authors in [6] express about the importance of ontologies to represent the knowledge found in corporate memory. Corporate memory consists of different types of knowledge, e.g., enterprise knowledge, knowledge about processes and products, clients and human resources, etc. Ontology-based knowledge contents are easy to locate, retain, access, and maintain. Ontologies provide the standard description of any domain, therefore, knowledge coming from different sources and different formats can be accumulated in a standard pattern. Such knowledge can be inferred easily by writing appropriate queries. Many authors emphasized on the need of ontology-based content management of FMEA knowledge. Some FMEA ontologies have been designed to support domain-specific knowledge management [7]. However, no one presented any solution to map existing FMEA knowledge to ontologies.

Motivation behind this piece of research is to develop a solution for populating FMEA ontology from existing worksheets in order to reutilize the existing knowledge for decision-making in future. For this, we evaluated an existing FMEA ontology [1] using OntoQA features. We found it rich with respect to schema, classes, and relationships among the classes. Then, we developed an algorithm, which extracts information from FMEA spread sheets and converts it to the FMEA ontology instances. All the procedure for managing these semantic contents is automatic. The overall knowledge management system has been evaluated by domain experts, and they were satisfied about its effectiveness.

This article is organized as follows. In Section 2, some related work is presented briefly, Section 3 is about the proposed work, Section 4 shows detailed evaluation of proposed work, and Section 5 finally concludes the discussion.

\section{Related Work}

In this section we will address different approaches, which have been seen for ontology-based organizational content management. Then, we will discuss about the ontology acquisition, tools, and techniques designed for it and their drawbacks.

\subsection{Ontology-Based Content Management Systems}

Ontology is a collection of concepts and relationship between those concepts. Knowledge base of an ontology stores different instances about the concepts and relationships defined in it. Authors in [8] addressed the importance of semantic content management. Sustainable enterprise relies on the effective management of its knowledge contents. Knowledge in an organization can be found scattered anywhere from white papers to web-accessible reports. There are numerous disadvantages 
of such scattered data. For example, it is difficult to analyze it in order to make conclusions, all the human efforts put to gather data are wasted, and it might lead to frauds and discrepancies. For a sustainable enterprise, it is a must to manage its knowledge repositories semantically so that it can be used in future for better decision-making. Authors believe that ontologies are a better way to manage enterprise contents semantically. Author in [9] focused on the issue of sustainability assessment and proposed an ontology-based knowledge management system as a solution.

Authors in [10] presented a domain ontology for sustainable development in economy. They addressed the environmental, economic, and social knowledge as the base of sustainable development. Authors in [11] show a close relationship between ontology and sustainability. Their emphasis is on the improvement of the knowledge acquired from the sustainability measures. They believe that ontologies model real-world phenomena, therefore, they are a better approach to understand the dimensions of sustainability. Authors in [12] presented an ontology-based knowledge base system for failure detection and prevention of cyber physical system. Such systems are equipped with the components for the detection and prevention of the system failure and their mitigation. Core component of such physical systems is a sensor whose failure leads to the failure of entire system. For the risk assessment of this sensor, authors utilized Failure Mode Effects and Critical Analysis (FMECA) approach. They stored their gathered knowledge in a knowledge base, which was constructed using ontology that helped deciding a mitigation action on identification of any hazard in the sensor. Authors in [6] presented an ontology-based content management system for a virtual organization. They provided a mechanism for ontology acquisition, storage, query, transformation, and information retrieval. They concluded that their searching and browsing experience was excellent with the help of ontology as it was focused on information retrieval and presentation.

Authors in [7] presented a conceptual framework for an ontology-based knowledge management system to preserve FMEA knowledge. This conceptual architecture was mainly based on domain-specific ontology to provide basic concepts of FMEA domain and relationship between them, a knowledge base to store instances, a query analyzer to ask knowledge base for a certain decision, and an inference engine to generate decisions from the knowledge base. This conceptual architecture can be seen in Figure 1.

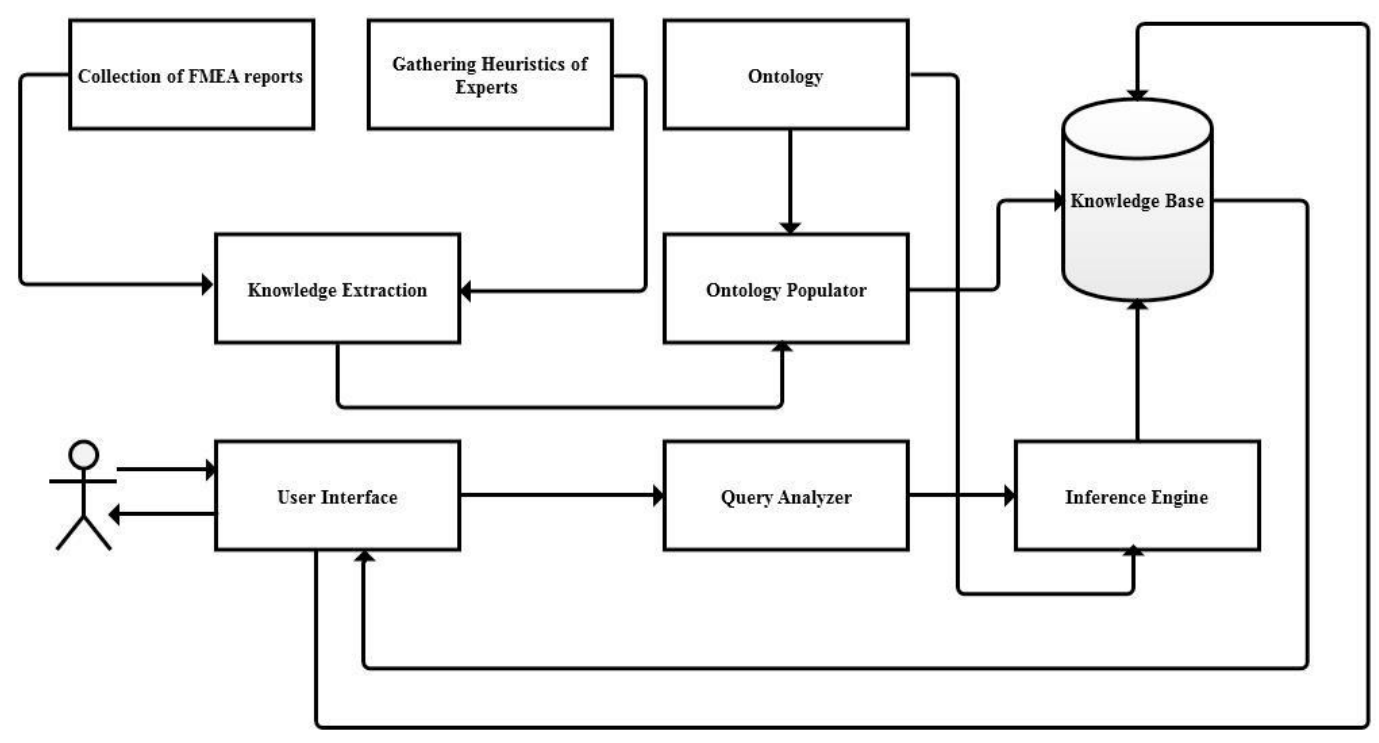

Figure 1. Conceptual architecture of ontology-based KBS for Failure Mode and Effect Analysis (FMEA) [7].

Later on, in [1], they provided implementation details of FMEA ontology and its query analyzer. They divided FMEA domain in six core concepts, e.g., FMEA, Process, Failure mode, RPN, Control 
method, and Mitigation action. They also provided a list of SPARQL queries to seek knowledge from the knowledge base. Overall performance of the system was excellent as it was answering all the queries properly. However, the knowledge extraction part of their conceptual frame work has not been addressed yet. It is rather difficult to develop a piece of software to extract information from conventional spreadsheets and store it in the knowledge base in the form of well-structured ontology instances because the information given in semi-structured documents does not contain explicit relationships. After FMEA ontology, this was another core part of that architecture without which the overall knowledge base system was ineffective. Their proposed FMEA ontology can be seen in Figure 2.

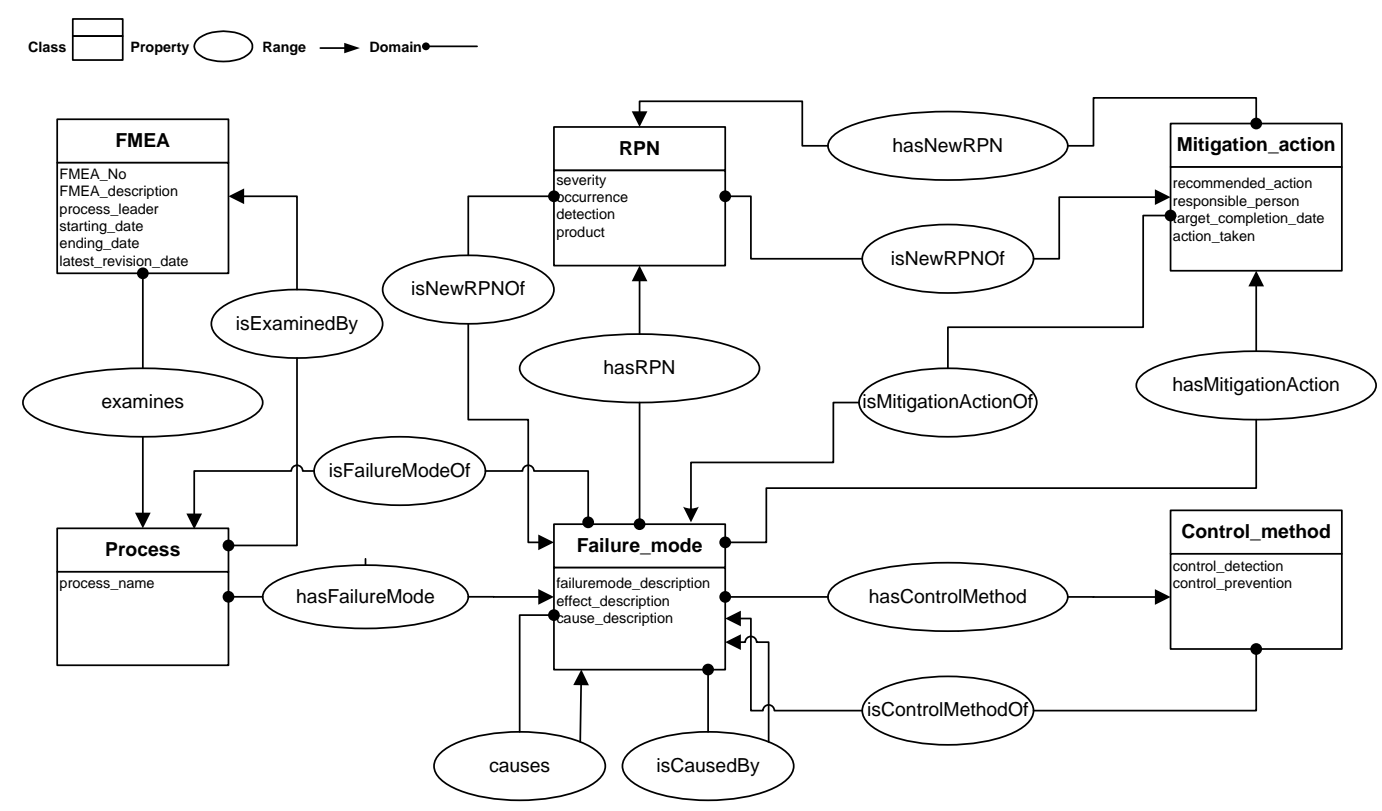

Figure 2. The FMEA ontology [1].

The work we have seen so far for ontology-based content management system just describes different studies to support ontology-based knowledge management systems. Core of these knowledge management systems is ontology, but no one addressed about the population of ontology and reusability of existing knowledge.

\subsection{Ontology Population Approaches}

While populating an existing ontology, its schema is not changed, however, only its knowledge base is enriched with new information [13]. According to [14]. "ontology population is the practice of adding concept and relation instances to an existing ontology knowledge base". It can be said, a process to build a knowledge base. It starts after building the conceptual model of the ontology and comprises different activities of adding new instances of concepts and relations into existing ontology. Ontology population phase requires a basic ontology, which will be populated, corpus, or corpora with new information, and a program to locate instances and relationships in corpus and populate them in ontology after converting into ontology instance language.

Manual instantiation of a knowledge base requires a lot of time, effort, and cost. Therefore, different techniques have been introduced to populate knowledge bases automatically orsemi-automatically. Some prominent approaches are discussed here. Authors in $[15,16]$ presented a system known as ArtEquAKT that automatically pulls out knowledge about artists available on the web, populates knowledge base with that knowledge, and further uses it to generate personalized biographies. It extracts Parts-of-Speech after syntactically analyzing web knowledge, and after that, uses semantic analysis to extract Named Entities and binary relationship between instances. It uses hand-built 
heuristics to handle with redundant instances. Another approach named "BOEMIE" [17] works on the similar patterns but uses machine learning to build heuristics that further help resolve redundancy. In this approach, machine learning is combined with ontology-based information extraction and an inference engine for extracting "primitive" concept instances from various modalities, which are converted to "composite" and more abstract concepts using abductive reasoning. Author in [18] proposed a system that applies statistical techniques to learn pattern extraction for binary relations in natural language corpora; it further uses this learning for automatically extracting instances of arbitrary binary relations from corpora. Authors in [19] presented an information extraction system that automatically populates knowledge base from soccer match reports from various semi-structured (tables) and unstructured (text, images, and image captions) information found on web. It identifies named entities using standard rule-based information extraction system, and uses mapping declarative rules to convert extracted named entities to semantic structures defined by the ontology. In [20], authors introduced a pattern-based method to populate subject ontology with the definitions of a domain glossary (domain of cultural heritage). This approach uses manually built lexico-syntactic patterns in order to extract instances of concepts from semi-structured corpora (Art and Architecture Thesaurus). Authors in [21] presented a semi-automated system that supports ontology population from both unstructured and semi-structured text. In this system, relation extraction is done by natural language processing techniques. Before assigning a candidate instance to a concept, its fitness value is calculated for each concept in the ontology using hierarchical syntactic information of the ontology schema. In the system presented by [22] a seed ontology is used to generate a domain ontology. Concept candidates are extracted using a general purpose named entity recognition system and lexico-syntactic patterns. These concept candidates are further filtered by calculating their statistical significance and according to the knowledge that can be acquired from available web sources. The system presented by [23] uses a bootstrapping approach. It extracts instances of relations from a given corpus, which are further validated by an ontology expert. Validated patterns are given to a patternlearner. After the completion of learner process, these induced patterns are used to extract new examples from unseen corpora, which are validated by a user. Authors in [14] proposed an approach, which is based on statistical, syntactic, and semantic techniques, for extracting instances for risk knowledge base using a generic ontology and collection of unstructured documents of the given domain. It uses Tree Tagger [24] to assign part of speech tags to the text found in the documents that carry information about probable risk. From these tagged words, all verbs are extracted and these verbs are supposed to be the relationships between instances of two concepts of the ontology. List of these verbs (relationships) is validated by a human expert. Further from each validated semantic relation in the given sentence of the corpus, a triplet is extracted (Segment ${ }_{-}$, Verb, and Segment ${ }_{R}$ ). These triplets are passed to a syntactic structure recognizer that uses hand-built rules to identify and generate instances (Instance- $\mathrm{L}$, Verb, and Instance-R) from candidate triplets. Authors in [25] used General Architecture of Text Engineering (GATE) for autoacquisition of a domain ontology from web-crawled Italian text. They also developed their required gazetteers of named entities. GATE is a good option while extracting information from unstructured documents where it is feasible to identify named entities and their relationship. Authors in [26] presented a deep learning-based NLP ontology population system to populate the Bimolecular Network Ontology (BNO) from textual data. The input to the system was unstructured text in the form of running sentences, which they tokenized before applying deep learning for information extraction. Then they used word2vec [27] algorithm to identify word embeddings with the help of a shallow neural network. After that, they populated BNO ontology with the help of a human expert who was responsible to evaluate each extracted instance by deep learning method. He had the responsibility to keep the ontology consistent.

All these techniques have been designed for unstructured text, which is in the form of sentences. These techniques identify named entities or other Parts-of-Speech and relationship between them, which can be found explicitly in the sentences. Therefore, none of these can be applied on FMEA worksheets in order to extract information and convert it into instances. 


\subsection{Comma Separated Values (CSV) to RDF Conversion Approaches}

We have to work on semi-structured CSV documents in order to extract instances for FMEA ontology. In this sort of information, the relations to bind information entities are not found, rather they are induced to form a triple of Resource Description Framework (RDF). Some applications that work on CSV to RDF conversion are given in Table 1.

Our desired features from a CSV to RDF convertor are given below, which are not found all together in any application given in Table 1.

1. A fully automated approach without human intervention.

2. Involvement of domain ontology for rapid mapping between CSV file and ontology language.

3. More than one instance could be defined from one row as it is not necessary for a row to have only a single instance.

4. Rows or columns can have multivalued attributes, which should be properly handled and have assigned relations with their correspondent instances correctly (without human intervention).

5. Empty attributes should also be handled.

6. Special characters, which can cause conflict with XML tags, should be converted properly.

Keeping these points in mind, we propose an algorithm that reads a CSV file, which contains semi-structured FMEA data, and this algorithm converts it to RDF instances. Some manually built patterns and tags are used in this algorithm, and it carefully embeds in them the information extracted from CSV file, which is later on written in OWL file as an RDF instance. This OWL file can be processed by any ontology editor, and it is also possible to execute SPARQL queries on it.

Table 1. Applications for conversion of Comma Separated Values (CSV) table information into Resource Description Framework (RDF) instances [28].

\begin{tabular}{|c|c|c|c|c|c|}
\hline Name & Data Source & $\begin{array}{l}\text { Mapping } \\
\text { Language }\end{array}$ & Mapping & $\begin{array}{l}\text { Requires Domain } \\
\text { Ontology }\end{array}$ & Uses GUI \\
\hline CSV2RDF4LOD & CSV & RDF & Manual & False & False \\
\hline Convert2RDF & Delimited text file & RDF/DAML & Manual & False & True \\
\hline $\begin{array}{l}\text { Google Refine } \\
\text { RDF Extension }\end{array}$ & CSV and XML & None & Semiautomatic & False & True \\
\hline Mapping Master & CSV & $\begin{array}{l}\text { Mapping } \\
\text { Master }\end{array}$ & GUI & False & True \\
\hline $\begin{array}{l}\text { OntoWiki CSV } \\
\text { Importer }\end{array}$ & CSV & $\begin{array}{c}\text { RDF Data Cube } \\
\text { Vocabulary }\end{array}$ & Semiautomatic & False & True \\
\hline RDF 123 & CSV & - & Manual & False & True \\
\hline T2LD & CSV & - & Automatic & False & False \\
\hline $\begin{array}{l}\text { TopBraid } \\
\text { Composer }\end{array}$ & CSV & SKOS & Semiautomatic & False & True \\
\hline $\begin{array}{l}\text { XLWrap: } \\
\text { Spreadsheet to } \\
\text { RDF }\end{array}$ & CSV & TriG Syntax & Manual & False & False \\
\hline
\end{tabular}

\section{Proposed Work}

Figure 3 represents the proposed ontology acquisition approach. We can see FMEA ontology and numerous FMEA spreadsheets, which have been prepared in the past manually by domain experts. Information in these spreadsheets is semi-structured that means concept instances are there but these sheets cannot provide any explicit relationship between them. Proposed algorithm is given in Section 3 . It extracts concept instances from FMEA spreadsheets. It is a must to have a spreadsheet prepared in the standard format as given in Figure 4. This algorithm induces relationship between concepts 
explicitly according to RDF format. Once a complete RDF instance is formed, it is added to existing FMEA ontology repository as an OWL-named individual, which can be queried to generate knowledge.

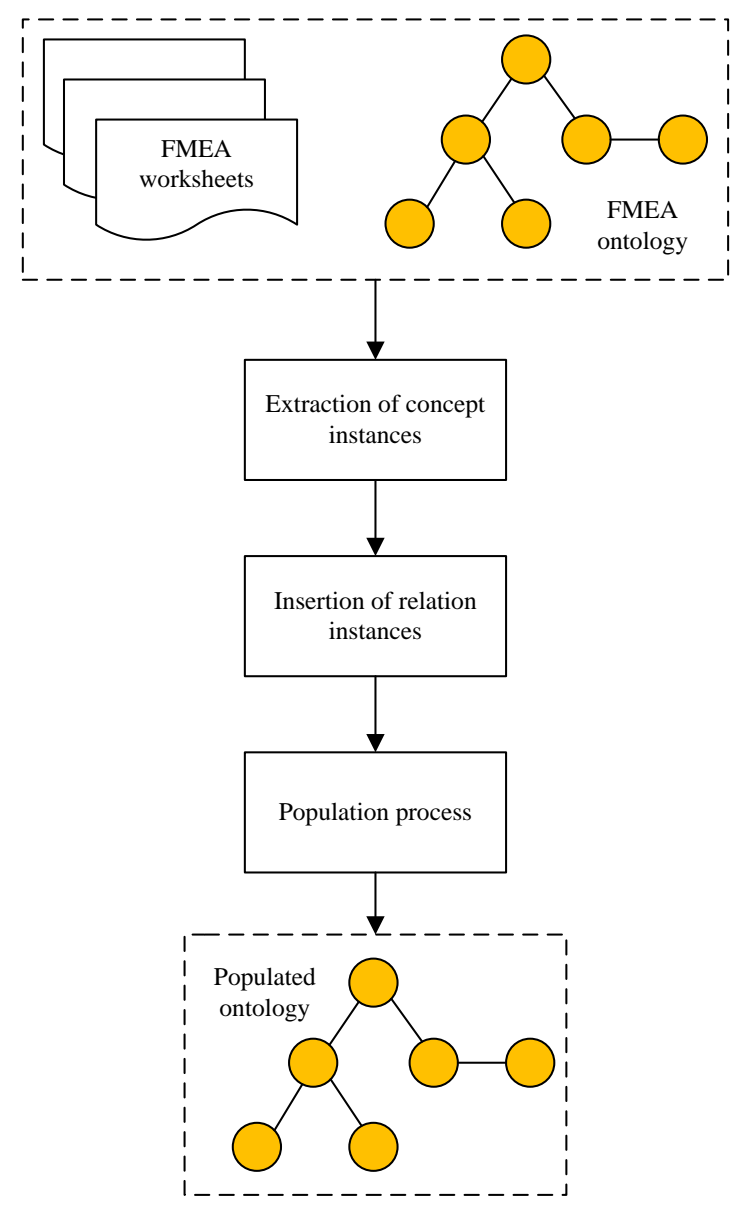

Figure 3. Automatic acquisition of FMEA ontology.

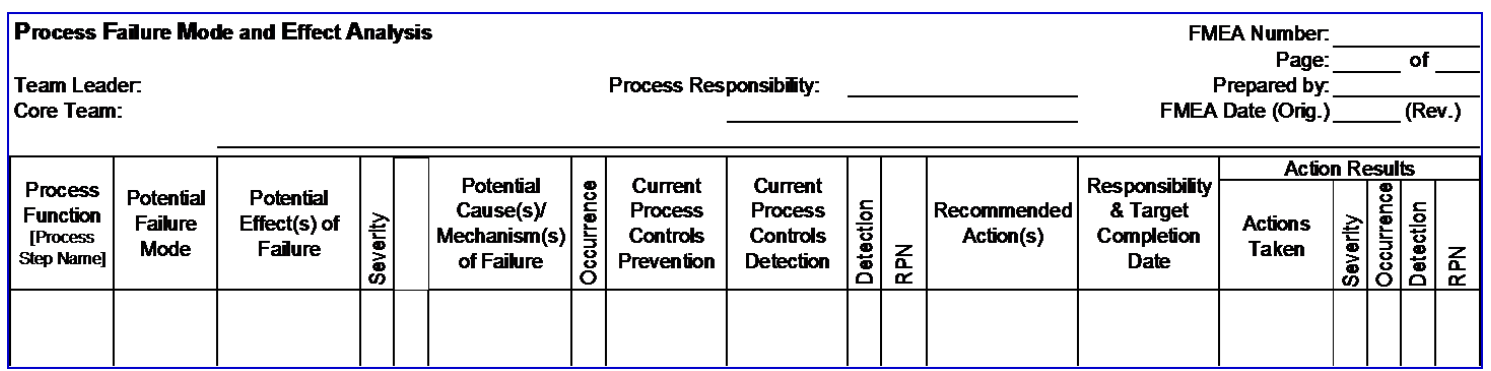

Figure 4. FMEA worksheet.

\section{Proposed Algorithm}

1. Load CSV file and read its contents in a 2D array named "FMEA table."

2. Read an existing OWL file and remove its end of file tag.

3. For each process in FMEA table:

a. For each failure mode

i. $\quad$ Read each effect

ii. Read each cause 
iii. Read each mitigation action

iv. Read RPN

b. Create a complete RDF instance including process description, failure mode, effect, cause, mitigation action, and RPN by inducing class relationships.

4. Embed this RDF instance as an OWL-named individual in existing OWL file.

5. Add end of file tag to the existing OWL file.

A sample FMEA worksheet is given in Figure 5. It contains FMEA information from an automotive domain process. Box 1 shows the output of the proposed algorithm when this sheet is passed to it as input.

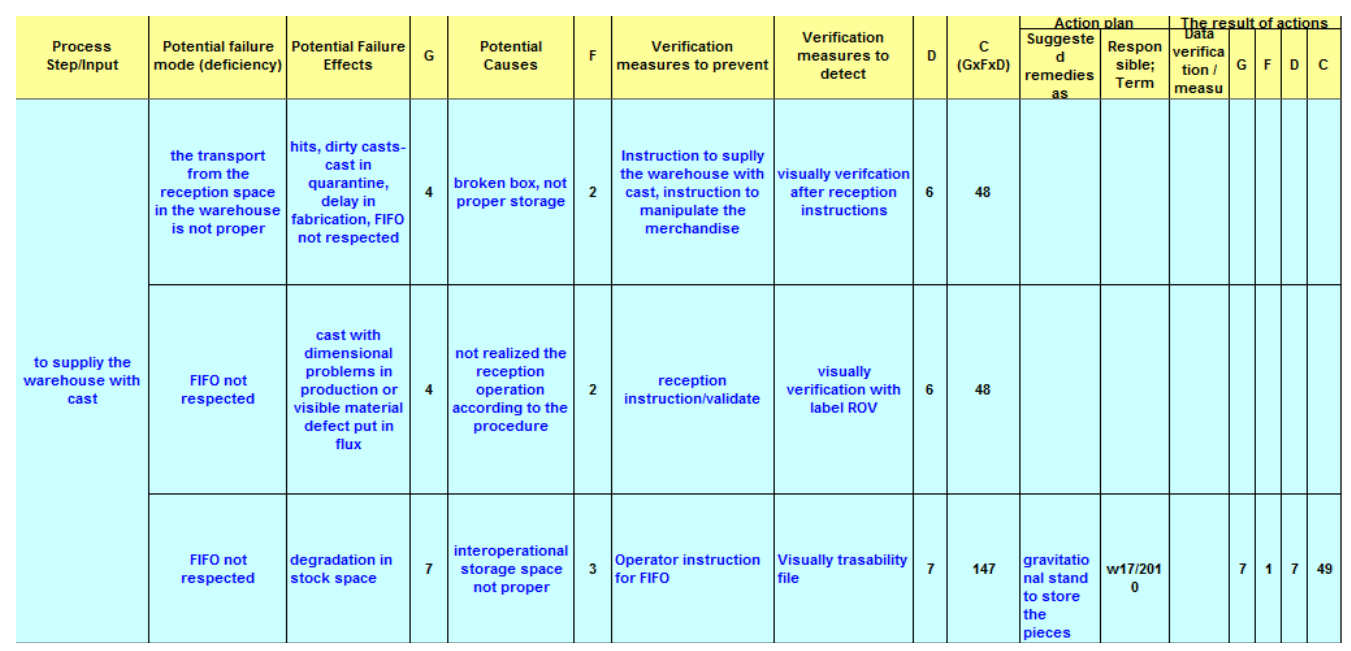

Figure 5. FMEA worksheet with sample data.

Listing 1. Output of the proposed algorithm with input given in Figure 5.

<owl:NamedIndividualrdf:about = "http://www.semanticweb.org/ontology\#Process199">

$<$ rdf:typerdf:resource = "http://www.semanticweb.org/ontology\#Process"/>

$<$ process_description $>$ FIFO not respected $<$ /process_description $>$

$<$ hasFailureModerdf:resource = "http://www.semanticweb.org/ontology\#FailureMode199" $/>$

$<$ hasFailureModerdf:resource = "http://www.semanticweb.org/rehman/ontology\#FailureMode200"/>

$<$ /owl:NamedIndividual $>$

<owl:NamedIndividualrdf:about = "http://www.semanticweb.org/ontology\#FailureMode199">

$<$ rdf:typerdf:resource = "http://www.semanticweb.org/ontology\#Failure_mode"/>

$<$ failuremode_description $>$ FIFO not respected $</$ failuremode_description $>$

$<$ isCausedByrdf:resource = "http://www.semanticweb.org/ontology\#Cause402"/>

<causes rdf:resource = "http://www.semanticweb.org/rehman/ontology\#Effect198"/>

$<$ owl:NamedIndividual $>$

<owl:NamedIndividualrdf:about = “http://www.semanticweb.org/ontology\#Effect198”>

$<$ rdf:typerdf:resource = "http://www.semanticweb.org/ontology\#Effect"/>

$<$ effect_description>cast with dimensional problems in production or visible material defect put in flux $</$ effect_description $>$ $</$ owl:NamedIndividual $>$

\section{Evaluation}

We evaluated this work from three aspects. First of all, we evaluated the FMEA ontology in order to know if its classes are disjoint to each other. This has been done by executing the consistency rules of Hermit reasoner. Disjointness ensures that the domain knowledge is explicit and ontology will provide only desired knowledge upon querying [29]. We also validated FMEA ontology for schema and 
relationship richness using OntoQA features. Second, we evaluated FMEA ontology after populating it with 1357 instances automatically. This evaluation was done by executing SPARQL queries on it and comparing their outcome with respective FMEA spreadsheets. Finally, it was evaluated from user's perspective. For this, some domain experts were invited to interact with it and answer a questionnaire. They were also given some supplementary information in the form of text. Details of these aspects are given below.

\subsection{Ontology Validation Using OntoQA Features}

OntoQA is a feature-based approach to evaluate and validate ontology schema and knowledge contents of ontology instances. Features proposed by this technique can be grouped into two features, i.e., "schema features" and "knowledge base" features.

\subsubsection{Schema Features}

Features in this category address the richness, width, depth, and inheritance of an ontology schema [30].

- $\quad$ Relationship Richness (RR)

"It is the ratio between the number of noninheritance relations $(\mathrm{P})$ and total number of relations in schema (sum of noninheritance relations $(\mathrm{P})$ and inheritance relations $(\mathrm{Q}))^{\prime \prime}$. The ontology with relationships of diverse nature is assumed to be more informative as compared to ontology that contains more inheritance relations.

$$
R R=|P| /(|P|+|Q|) .
$$

In case of FMEA ontology, it has 2 inheritance relationships and 6 noninheritance relationships, therefore RR of FMEA ontology is 0.25 ; greater number of noninheritance relationships indicates that FMEA ontology is adequately informative.

$$
\begin{aligned}
R R= & |2| /(|2|+|6|) \\
& =0.25 .
\end{aligned}
$$

\subsubsection{Attribute Richness (AR)}

"It is the ratio between total number of attributes (attribute) of all classes and the number of classes (c) in ontology". Higher value of attribute richness indicates that ontology conveys more knowledge.

$$
\mathrm{AR}=\mid \text { attribute }|/| \mathrm{C} \mid
$$

FMEA ontology is composed of 8 classes, whereas all these classes collectively have 20 attributes, therefore, AR of FMEA ontology is 2.5 .

$$
\begin{aligned}
\mathrm{AR} & =|20| /|8| \\
& =2.5 .
\end{aligned}
$$

\subsubsection{Knowledge Base Features}

These are the features that describe the knowledge base and distribution of knowledge in each class of schema. Distribution of knowledge over ontology classes indicates the effectiveness of real-world knowledge conveyed to domain users.

- $\quad$ Class Richness (CR) 
"It is the percentage ratio between nonempty classes $\left(C^{\prime}\right)$ and total number of classes $(C)$ in an ontology schema." Higher value of CR indicates that knowledge base has adequate knowledge to exemplify knowledge in the schema.

$$
\mathrm{CR}=\left(\left|\mathrm{C}^{\prime}\right| /|\mathrm{C}|\right) * 100 \text {. }
$$

FMEA ontology does not contain any empty classes, therefore, it has $100 \%$ class richness.

$$
\begin{aligned}
\mathrm{CR}= & (|8| /|8|) * 100 \\
= & 100 .
\end{aligned}
$$

\section{- Class Connectivity (Conn)}

This feature helps to identify the focal classes in the ontology by calculating "the number of connections of instances of one class to the instances of the other classes in ontology". Class connectivity of FMEA ontology is shown in Figure 6. Total number of relationship instances was 1726.

$$
\text { Conn }\left(C_{i}\right)=\mid \text { No_Ins_Rel }\left(C_{i}\right) \mid \text {. }
$$

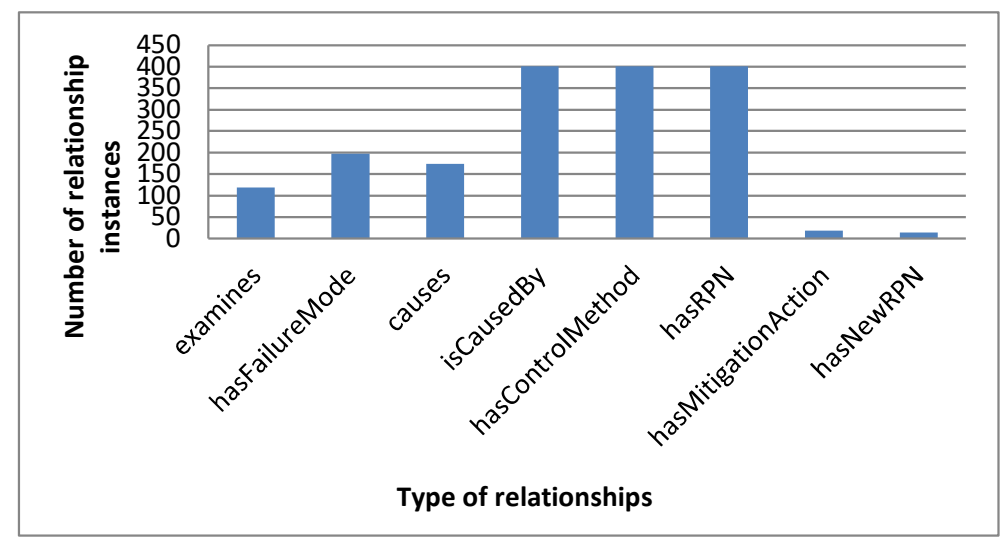

Figure 6. FMEA ontology class connectivity.

- $\quad$ Class Importance (Imp)

Class importance is calculated on the basis of number of instances belonging to a specific class. "It is the percentage ratio between the number of instances of a particular class $\left(\mathrm{C}_{\mathrm{i}}\right)$ to the total number of instances in the knowledge base $\left(C_{n}\right)^{\prime \prime}$. Class importance of FMEA ontology is shown in Figure 7.

$$
\operatorname{Imp}\left(\mathrm{C}_{\mathrm{i}}\right)=\left|\mathrm{No} \_I n s \_\left(\mathrm{C}_{\mathrm{i}}\right) / /\right| \mathrm{No} \_I n s \_\mathrm{Kb}\left(\mathrm{C}_{\mathrm{n}}\right) \mid \text {. }
$$

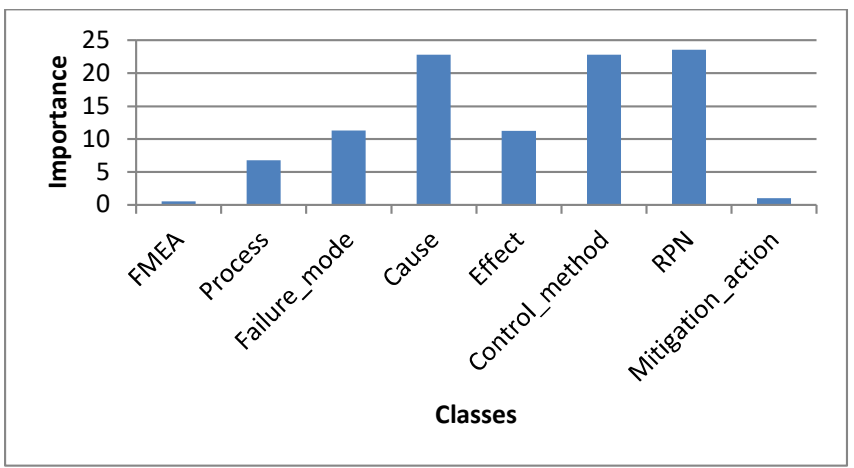

Figure 7. Importance of different classes in FMEA ontology. 
- $\quad$ Relationship Richness $\left(\mathrm{RR}_{\mathrm{kb}}\right)$

"It is the percentage comparison of the number of relations being used by instances actually $P\left(I_{i}, I_{j}\right)$ and number of relationships defined for a class in schema level $P\left(C_{i}, C_{j}\right)^{\prime \prime}$.

$$
\begin{aligned}
\mathrm{RR}_{\mathrm{kb}}=\left(\mathrm{P}\left(\mathrm{I}_{\mathrm{i}}, \mathrm{I}_{\mathrm{j}}\right) / \mathrm{P}\left(\mathrm{C}_{\mathrm{i}}, \mathrm{C}_{\mathrm{j}}\right)\right) * 100 \\
\mathrm{RR}_{\mathrm{kb}}=(8 / 8) * 100 \\
=100 .
\end{aligned}
$$

In FMEA, ontology schema 8 relationships have been defined, and all these relationships have been used in knowledge base; therefore, relationship richness of FMEA knowledge base is 100\%.

\subsection{Evaluation of the Populated Ontology by Querying It}

We tested the populated ontology for all 1357 instances using different queries. We matched the output of each query with the spreadsheets' data, and we found it correctly populated. A query is given in Box 2 to display causes, effects, and recommendations for a specific failure mode.

Listing 2. SPARQL query to display causes, effects, and recommendations for a failure mode.

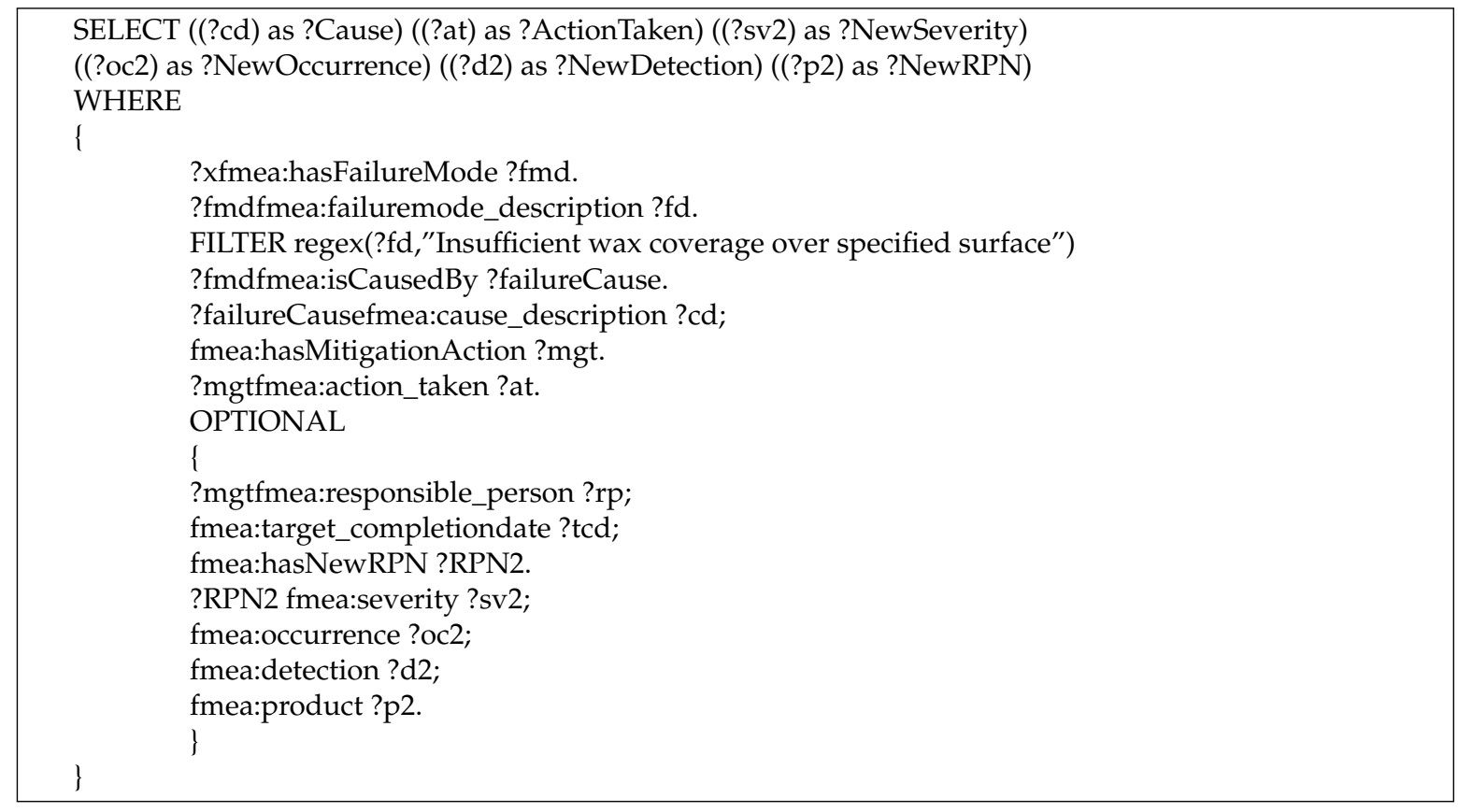

Output of this query is shown in Figure 8.

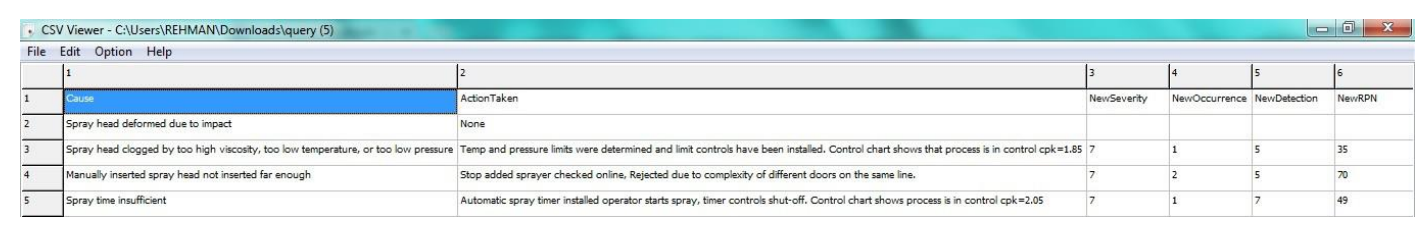

Figure 8. Causes and recommendations for a specific failure mode in Comma Separated Values viewer.

\subsection{Evaluation in the Light of Expert's Opinion}

While evaluating a KMS, experts' opinion and feedback of its direct users really matters. Ideal way to collect this feedback is to deploy the KMS in a best suited organization and invite experts and 
concerned employees to interact with it so that they can practically experience how new technology effects their everyday work, how much time they can save from such systems, what level of ease or difficulty they face while using a new system, and, overall, how it can effect organizational benefits. Although it was not possible, but as an alternative, we conducted a questionnaire-based survey by providing respondents with supplementary information about this KMS and its working. They were also given the opportunity to query it. We distributed a questionnaire (given in Appendix A) in industrial and academia experts who deal with knowledge management, quality engineering, and risk management.

In questionnaire, respondents have been asked to express their level of expertise in terms of their experience in years and practice of FMEA for risk assessment. They were given some choices about their concerned areas. Figure 9 shows that majority of them were from quality management, whereas Figures 10 and 11 depict their experience and expertise, respectively.

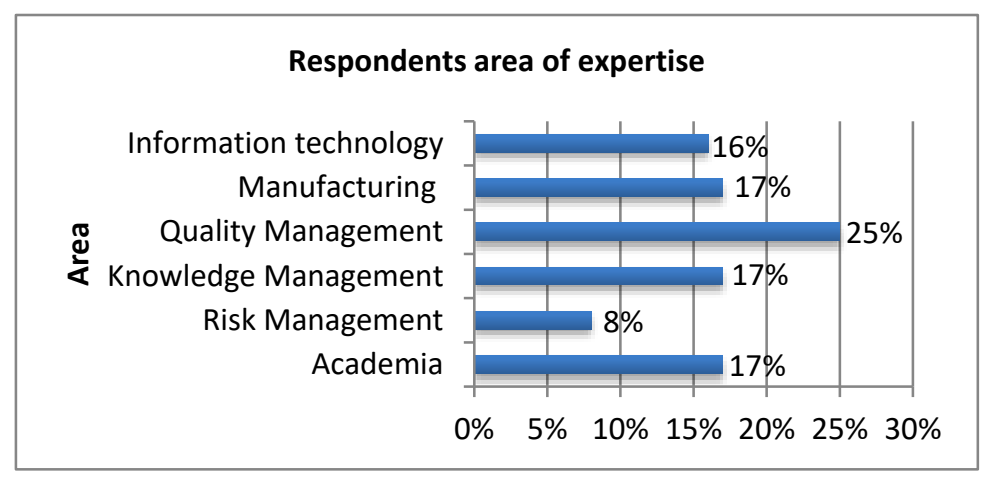

Figure 9. Area of expertise of survey respondents.

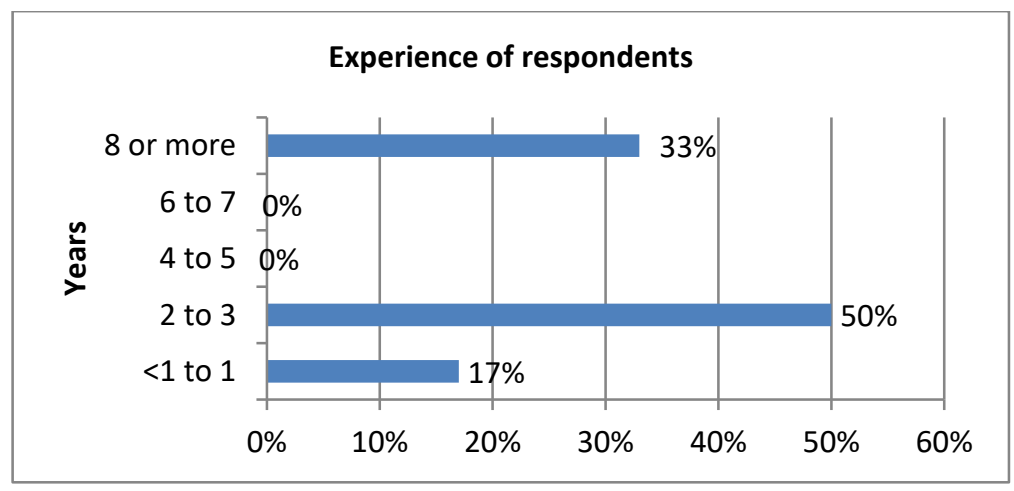

Figure 10. Experience of survey respondents in years.

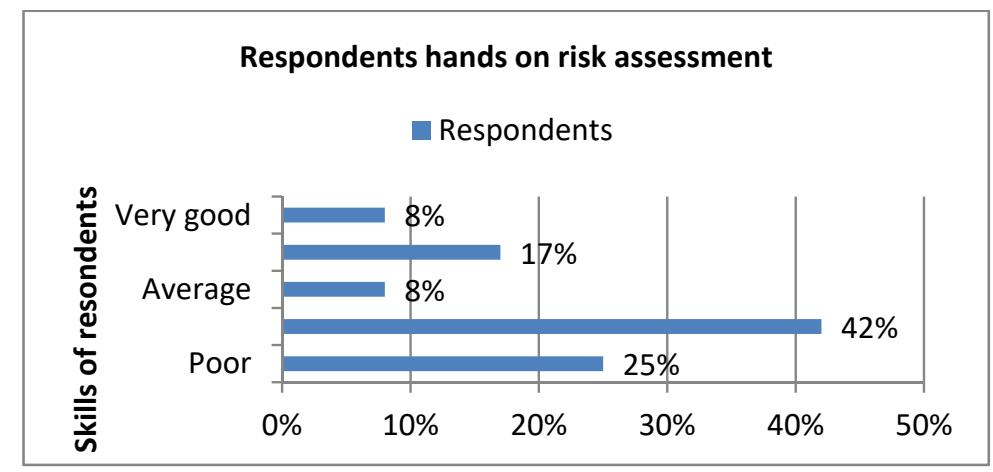

Figure 11. Respondents expertise in risk management particularly in FMEA. 
Respondents were requested to compare traditional process of FMEA with knowledge-based FMEA using parameters of time, cost effectiveness, and ease of use. According to their response, both of these FMEA procedures have been compared. For example, respondents were given 5 options for the question to compare the cost effectiveness of each option, which had a rating score associated with it: (i) traditional FMEA method is slightly cost effective than knowledge-based FMEA method $=1$, (ii) traditional FMEA method is more cost effective than knowledge-based FMEA method = 2, (iii) both have the same impact $=0$, (iv) knowledge-based FMEA method is slightly cost effective than traditional FMEA method $=1$, and (v) knowledge-based FMEA method is more cost effective than traditional FMEA method $=2$. Average of options favoring traditional FMEA procedure is calculated by adding their magnitudes and dividing the sum with sample size $n=12$. Similarly, average of options favoring knowledge-based FMEA procedure is calculated by adding their magnitude and dividing the sum with sample size $n=12$. Similar calculations have been carried out for the responses of time effectiveness and ease of use. Comparison of the results is shown in Figure 12 that clearly depicts advantages of knowledge-based FMEA process over traditional FMEA process.

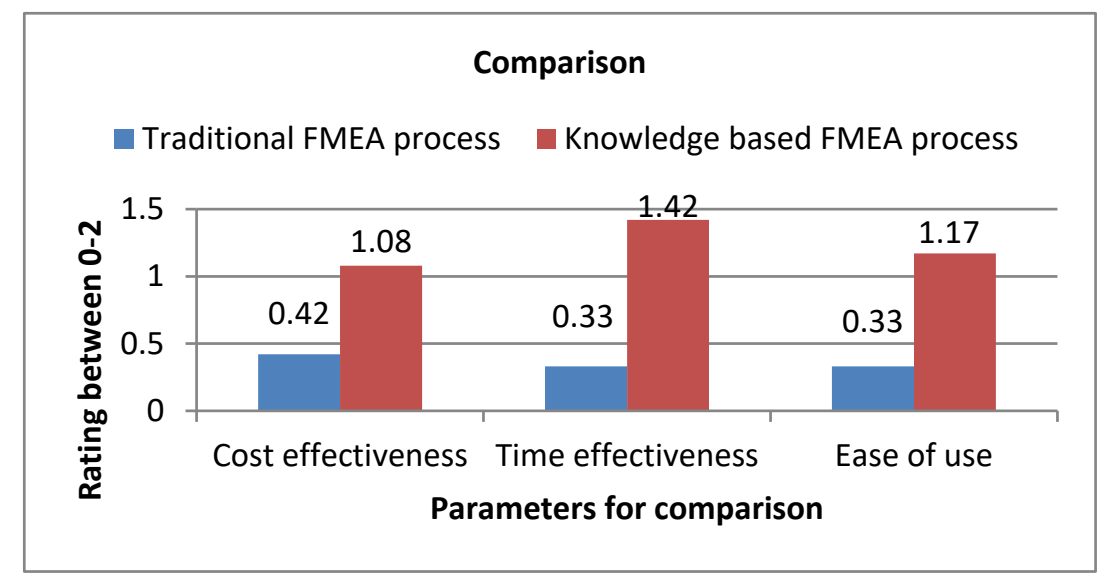

Figure 12. Traditional FMEA process vs. knowledge-based FMEA process.

Respondents have also been asked to give their opinion about the impact of proposed system on its novice users, organizational culture, performance, and further record keeping of FMEA knowledge. Results of their opinions for these questions are shown in Figure 13 to Figure 14.

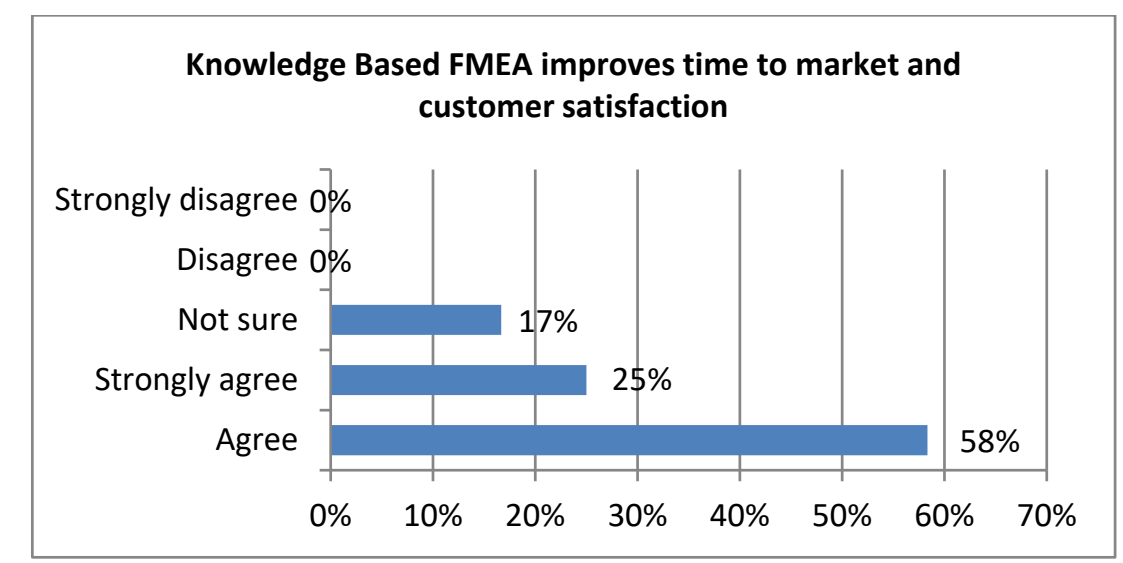

Figure 13. Respondent's opinion about knowledge-based FMEA impact on customer satisfaction. 


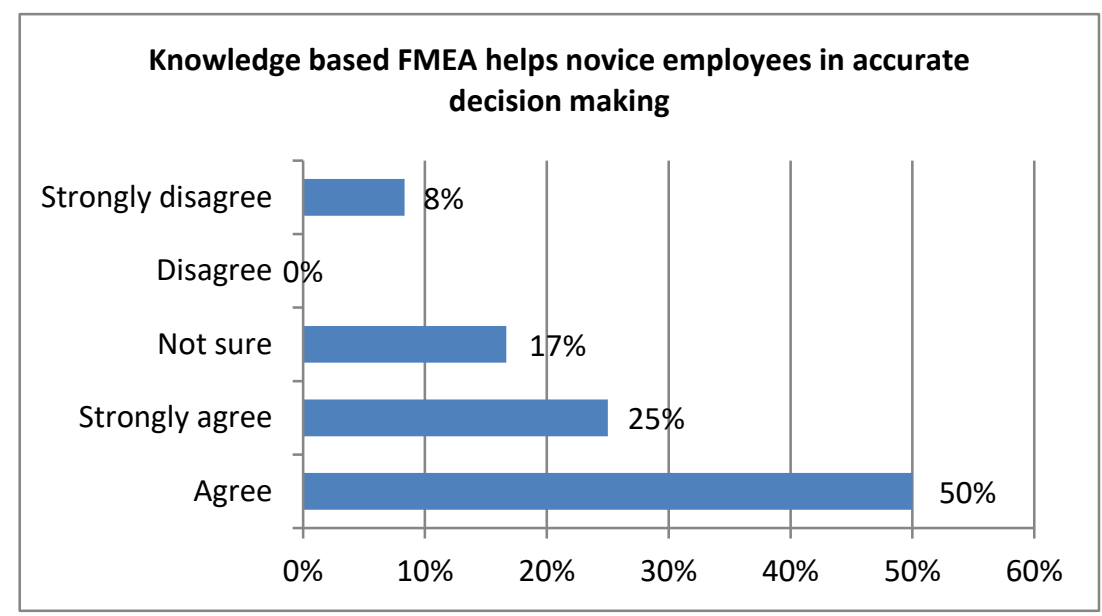

(a)

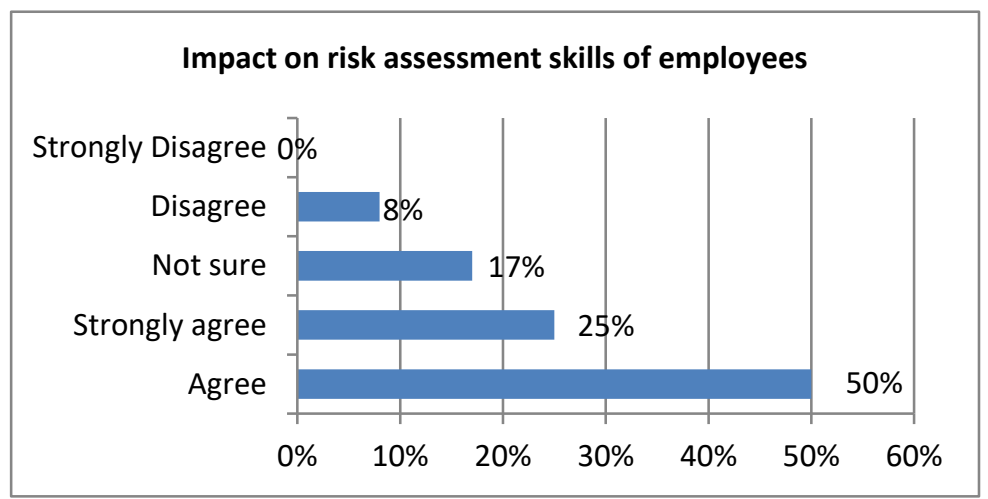

(b)

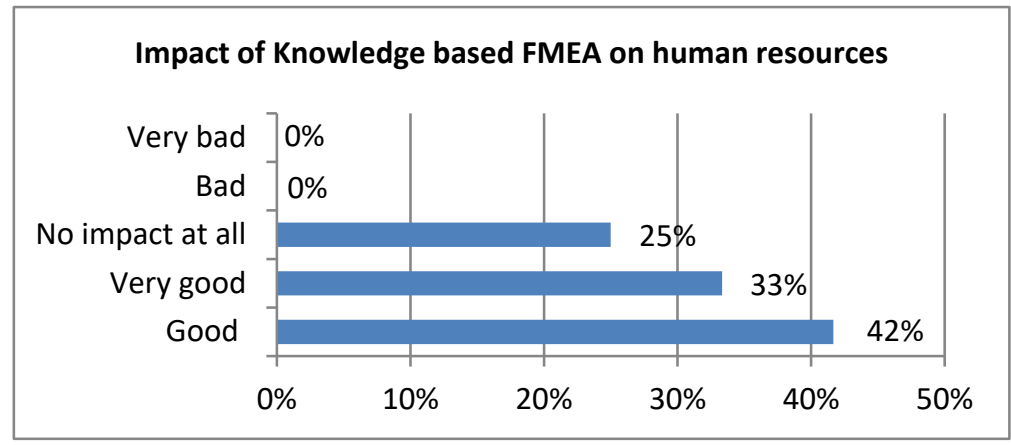

(c)

Figure 14. (a) Respondents opinion about knowledge-based FMEA's impact on employees' decision-making. (b) Respondents opinion about knowledge-based FMEA impact on risk assessment skills of employees. (c) Respondents opinion about knowledge-based FMEA impact on human resources.

Figure 13 shows that 58\% respondents agree and 25\% strongly agree that knowledge-based FMEA will accelerate time to market and as it will help making decisions on the basis of past experience, therefore, they will lead to more robust product or process that will, in turn, improve customer satisfaction.

Figure 14 a shows that 50\% respondents agree and 25\% strongly agree that knowledge-based FMEA helps novice employees in making accurate decisions. We believe that such systems can be very helpful in training internees and novice employees in an organization. They are the people who can give 
expert like performance for low wages if they are given the heuristics of experts in an understandable format. This characteristic of KBS makes them attractive for scarce-resourced organizations.

Past experiences are also helpful for experts as they can assess a failure in a far better way if they could know about its impact in the light of the knowledge of their fellow experts and impacts of their proposed mitigation actions. According to our survey, 50\% people agree and 25\% strongly agree that it polishes risk assessment skills of employees as shown in Figure 14b.

As shown in Figures 14c and 15a, most of the respondents agree about the positive impacts on knowledge-based FMEA on organizational environment. These systems boost learning environment inside an organization, it also grooms confidence level of individual on their own capabilities of decision-making. Following the footsteps of domain legends, they can build their level of expertise in a relatively shorter time.

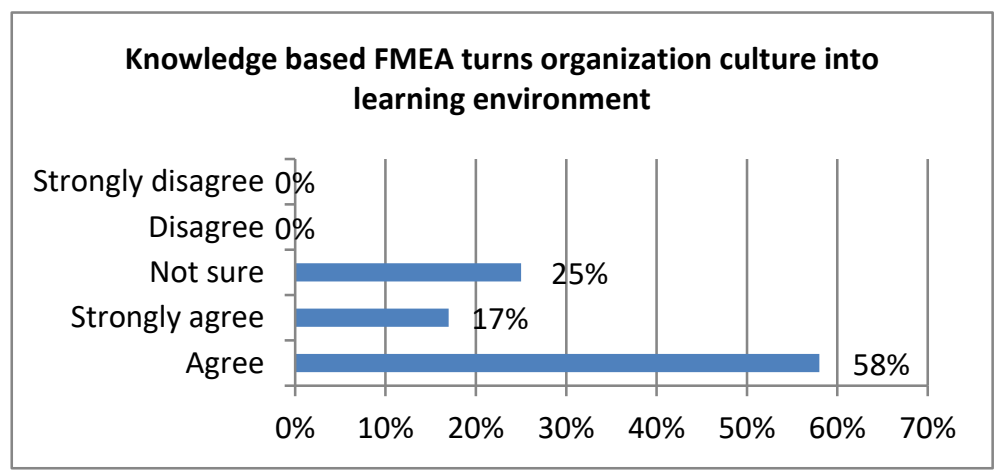

(a)

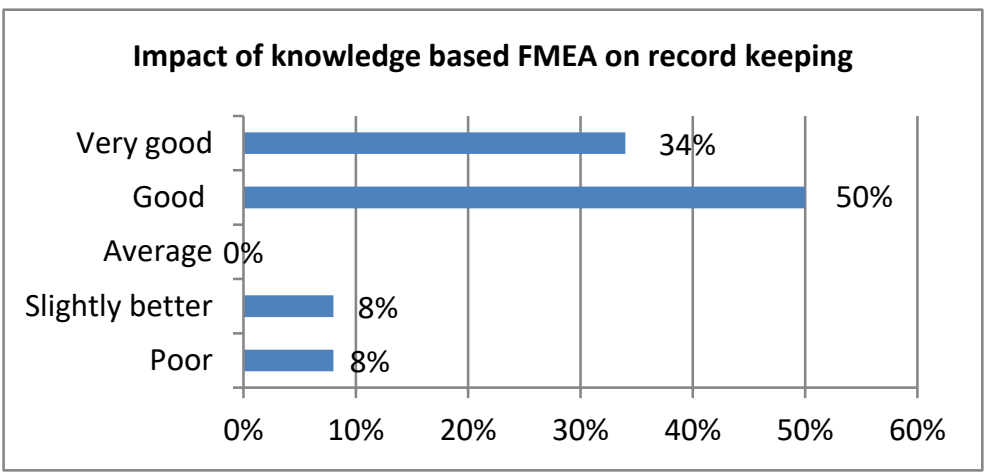

(b)

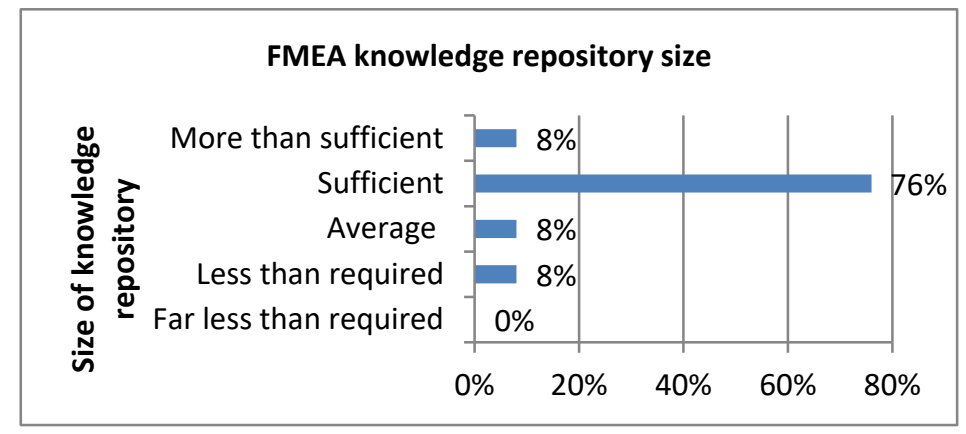

(c)

Figure 15. (a) Respondents' opinion about knowledge-based FMEA impact on organizational environment. (b) Respondents' opinion about knowledge-based FMEA impact on risk record keeping. (c) Respondents' opinion about size of FMEA knowledge repository. 
Figure 15b shows how survey respondents' overview the record keeping services of the proposed system. In fact, this system provides best services of record keeping as it is accompanied by a fully automatic parser that easily integrates new records into repository and keeps it consistent and up to date.

In case of KBS, the size of knowledge repository matters a lot. We have about 1357 instances of FMEA from automotive domain in FMEA repository. In addition, respondents have been asked about whether the instances are sufficient or not and their response is shown in Figure 15c. According to Figure 16 respondents were interacting with the very first ontology based content management system for FMEA.

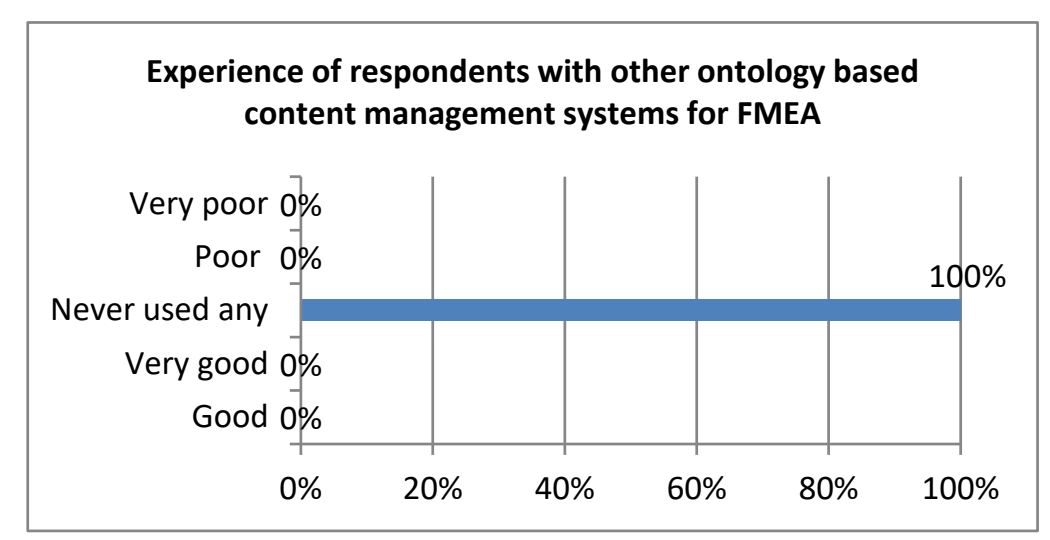

Figure 16. Experience of respondents about other ontology-based content management system for FMEA.

We really wished to compare our KMS with any other available KMS for FMEA. For this purpose, we asked respondents to name that if they have already used it and let us know where our proposed system stands as compared to that. However, unfortunately, none of them had used such a system ever before.

\subsection{Comparison with Existing Ontology-Based KMS for FMEA}

Different ontology-based KMS are shown in Table 2, which have been implemented to support reusability of FMEA knowledge. For the ontology-based KMS, it is not only important to model the domain knowledge in a proper way by choosing appropriate methodology but also it is equally important to pay attention towards the acquisition of ontology knowledge base and infer new knowledge through it. In Table 2, it can be seen how these approaches modeled domain knowledge. It can also be seen that none of them considered effective acquisition of knowledge base. Although ontology provides same knowledge with either manual or automatically acquired knowledge base, time or effort spent to populate ontology manually fades entire charm of a KMS. In our work, we paid special attention towards this aspect, and we provided an algorithm to extract FMEA information from worksheets and populate it to knowledge base. Although our algorithm is domain specific and extracts information given in a specified pattern, it saves time and effort to populate ontology manually and works with optimal precision. 
Table 2. Ontology-based knowledge management systems to support Failure Mode and Effect Analysis (FMEA).

\begin{tabular}{|c|c|c|c|c|c|}
\hline Approach & Domain & $\begin{array}{c}\text { Ontology } \\
\text { Methodology }\end{array}$ & Tools & $\begin{array}{l}\text { Knowledge } \\
\text { Base } \\
\text { Acquisition }\end{array}$ & $\begin{array}{c}\text { Size of } \\
\text { Knowledge } \\
\text { Base }\end{array}$ \\
\hline$[31]$ & Unknown & $\begin{array}{c}\text { TOVE } \\
\text { methodology }\end{array}$ & F-logic & Manual & Not mentioned \\
\hline [32] & $\begin{array}{c}\text { Tehran Subway } \\
\text { Project }\end{array}$ & Not mentioned & $\begin{array}{c}\text { Protégé, } \\
\text { ARP Servlet }\end{array}$ & Not mentioned & Not mentioned \\
\hline [33] & $\begin{array}{l}\text { Company } \\
\text { project that } \\
\text { produces cage } \\
\text { component in } \\
\text { bearings }\end{array}$ & Methontology & $\begin{array}{l}\text { Protégé, } \\
\text { RacerPro }\end{array}$ & Manual & Not mentioned \\
\hline $\begin{array}{l}\text { Proposed } \\
\text { approach }\end{array}$ & $\begin{array}{l}\text { Automotive } \\
\text { processes }\end{array}$ & $\begin{array}{c}\text { Hybrid } \\
\text { (Methontology } \\
\text { and OTK) }\end{array}$ & $\begin{array}{c}\text { Protégé, } \\
\text { Hermit, Python }\end{array}$ & Automatic & 1357 instances \\
\hline
\end{tabular}

We can conclude that FMEA ontology used for automatic acquisition was rich with respect to schema, relationships and number of instances. From users' perspective that actually interacted with this KMS it was obvious that it is easy to use, and on each query, it provided real knowledge from automatically acquired knowledge base.

\section{Conclusions}

In this article, we discussed about the importance of semantic content management of FMEA knowledge. Organizations spend cost, effort, and time to prepare this knowledge but it resides in spreadsheets and cannot be reused. Ontology-based content management systems has been proposed by many authors as discussed in Section 2.1 but acquiring ontology from existing FMEA sheets has not been addressed yet. Moreover, available ontology population approaches are not smart enough to map all the knowledge correctly to ontology. Therefore, we developed an algorithm for this purpose, which extracts knowledge from standard FMEA worksheets and converts it to RDF instances. This algorithm mapped 1357 instances to FMEA ontology with 100\% accuracy. For this experiment, we selected an existing ontology presented by authors in [1] and evaluated it for ontoQA features. This ontology was the part of a complete knowledge management system. This was presented to domain users for evaluating its effectiveness. They agreed upon that knowledge repository was sufficient enough to answer all their queries. Moreover, they concluded that this system was very helpful for novice employees in making expert-like decisions, record keeping, and improving time to market.

Author Contributions: Conceptualization, Z.R.; methodology, Z.R.; software, Z.R.; validation, C.V.K. and S.N.; formal analysis, C.V.K.; investigation, Z.R.; resources, C.V.K.; data curation, C.V.K.; writing-original draft preparation, Z.R.; writing—review and editing, F.J; visualization, M.W.; supervision, C.V.K.; project administration, C.V.K.; funding acquisition, C.V.K. All authors have read and agreed to the published version of the manuscript.

Funding: This work has been financed from Lucian Blaga University of Sibiu, The Research centre for sustainable products and processes and Hasso Plattner Foundation research action LBUS-RRC-2020-01.

Conflicts of Interest: The authors declare no conflict of interest.

\section{Appendix A}

The survey form given in Table A1 has been filled by those people who actually interacted with the system and was having the knowledge of conducting FMEA process manually. 
Table A1. Questionnaire 1.

1. Does our proposed Knowledge based FMEA sound cost effective as compared to conventional FMEA method?
(a) Traditional FMEA method is slightly cost effective than knowledge based FMEA method
(b) Traditional FMEA method is more cost effective than knowledge based FMEA method
(c) Both have the same impact
(d) Knowledge based FMEA method is slightly cost effective than traditional FMEA method
(e) Knowledge based FMEA method is more cost effective than traditional FMEA method

2 Which one is more time effective?
(a) Traditional FMEA method is slightly time effective than knowledge based FMEA method
(b) Traditional FMEA method is more time effective than knowledge based FMEA method
(c) Both have the same impact
(d) Knowledge based FMEA method is slightly time effective than traditional FMEA method
(e) Knowledge based FMEA method is more time effective than traditional FMEA method

3 Can it help an organization improve time to market and customer satisfaction?
(a) Agree
(b) Strongly agree
(c) Not sure
(d) Disagree
(e) Strongly disagree

$4 \quad$ Can it help a novice employee make an expert like decisions for risk assessment?
(a) Agree
(b) Strongly agree
(c) Not sure
(d) Disagree
(e) Strongly disagree

5 If this system is implemented in an organization how would be its impact on human resources there?
(a) Good
(b) Very good
(c) No impact at all
(d) $\mathrm{Bad}$
(e) Very bad

Please give here your comments to support your answer for question 5:

6 Do you think that it will turn the organizational culture into learning environment where novice users have heuristics of experts at a distance of a single click?
(a) Agree
(b) Strongly agree
(c) Not sure
(d) Disagree
(e) Strongly disagree

$7 \quad$ Will it improve risk assessment skills of employees?
(a) Agree
(b) Strongly agree
(c) Not sure
(d) Disagree
(e) Strongly disagree 
Table A1. Cont.

8 Would it be easier to deploy it in an organization and train employees and make them switch on it from tradition methods?
(a) Traditional methods are slightly convenient to deploy and learn
(b) Traditional methods are more convenient to deploy and learn
(c) Both have equal complexity
(d) Proposed method sounds slightly convenient to deploy and learn
(e) Proposed method sounds more convenient to deploy and learn

9 What would be its effect if it comes to FMEA record keeping in an organization?
(a) Poor
(b) Slightly better
(c) Average
(d) Good
(e) Very good

10 Do you think FMEA knowledge repository for automotive process domain with 1357 instances distributed over failure mode, its cause(s), effect(s), control method(s), and mitigation action(s) is rich enough to assist a novice employee in making an effective decision?
(a) Far less than required
(b) Less than required
(c) Average
(d) Sufficient
(e) More than sufficient

11 Have you ever tried an Information Technology based Knowledge management system for FMEA? Please name it here system stand if compared to that? and let us know where does our proposed
(a) Good
(b) Very good
(c) Never used any
(d) Poor
(e) Very poor

12 Please state if you see any shortfalls in our proposed system:

13 Please state your opinion about technical advantages of our proposed system for an organization:

14 Which of the following category (or more than one) best describe your job affiliation?
(a) Academia
(b) Risk management
(c) Quality management
(d) Knowledge management
(e) Manufacturing
(f) Information technology

15 How long you have been working in your related field?
(a) Less than 1 year
(b) 2-3 years
(c) 4-5 years
(d) 6-7 years
(e) 8 or more years 
Table A1. Cont.

16 How skillful you are regarding implementation of FMEA for risk assessment?
(a) Poor
(b) Basic
(c) Average
(d) Good
(e) Very good

\section{References}

1. Rehman, Z.; Kifor, C. An Ontology to Support Semantic Management of FMEA Knowledge. Int. J. Comput. Commun. Control 2016, 11,507-521. [CrossRef]

2. Wu, J.; Lo, M.F.; Ng, A.W. Knowledge Management and Sustainable Developmen. In Encyclopedia of Sustainability in Higher Education; Leal, F.W., Ed.; Springer International Publishing: Cham, Swizerland, 2018; pp. 1-9.

3. Lai, F.W.; Samad, F. Enterprise Risk Management Framework and Empirical Determinants of Its Implementation. In Proceedings of the International Conference on Business and Economics Research, Kuala Lumpur, Malaysia, 26-28 November 2010.

4. Nguyen, T.L.; Shu, M.H.; Hsu, B.M. Extended FMEA for Sustainable Manufacturing: An Empirical Study in Non-Wooven Fabric Industry. Sustainability 2016, 8, 939. [CrossRef]

5. Jaroslava, K.; Ivana, R. Enterprise Content Management Implementation and Risk. Acta Univ. Agric. Silvic. Mendel. Brun. 2015, 63, 1687-1695.

6. Mika, P.; Iosif, V.; Sure, Y.; Akkermans, H. Ontology-based Content Management in a Virtual Organization. In Handbook on Ontologies. International Handbooks on Information Systems; Springer: Berlin/Heidelberg, Germany, 2004.

7. Rehman, Z.; Kifor, S. A Conceptual Architecture of Ontology Based KM System for Failure Mode and Effects Analysis. Int. J. Comput. Commun. Contol 2014, 9, 463-470. [CrossRef]

8. Sheth, A.; Fisher, M. Semantic Enterprise Content Management; CRC Press: Boca Raton, FL, USA, 2004.

9. Konys, A. An Ontology-Based Knowledge Modelling for a Sustainability Assessment Domain. Sustainability 2018, 10, 300. [CrossRef]

10. Ivanova, A.; Deliyska, B.; Todorov, V. Domain ontology of sustainable development in economy. In AIP Conference Proceedings; AIP Publisher LLC.: Melville, NY, USA, 2018.

11. Wijesooriya, C.; Heales, J.; McCoy, S. Multi-Dimensional Views for Sustainability: Ontological approach. In Proceedings of the Twenty-first Americas Conference on Information Systems, Fajardo, Puerto Rico, 13-15 August 2015.

12. Ali, N.; Hong, J.-E. Failure Detection and Prevention for Cyber-Physical Systems Using Ontology-Based Knowledge Base. Computers. 2018, 7, 68. [CrossRef]

13. Paliouras, G.; Karkaletsis, V.; Paliouras, G.; Krithara, A.; Zavitsanos, E. Ontology Population and Enrichment: State of the Art. In Knowledge-Driven Multimedia Information Extraction and Ontology Evolution, Lecture Notes in Computer Science; Springer: Berlin/Heidelberg, Germany, 2011; pp. 134-166.

14. Makki, J.; Alquier, A.-M.; Prince, V. Ontology Population via NLP Techniques in Risk Management. Int. J. Hum. Soc. Sci. 2009, III, 212-217.

15. Kim, S.; Alani, H.; Hall, W.; Lewis, P.; Millard, D.; Shadbolt, N.; Weal, M. Artequakt: Generating Tailored Biographies from Automatically Annotated Fragments from the Web. In Proceedings of Workshop on Semantic Authoring, Annotation \& Knowledge Markup (SAAKM'02), the 15th European Conference on Artificial Intelligence, Lyon, France, 21-26 July 2002; pp. 1-6.

16. Alani, H.; Kim, S.; Millard, D.E.; Weal, M.J.; Lewis, P.H.; Hall, W.; Shadbolt, N.R. Automatic Extraction of Knowledge from Web Documents. In Proceedings of the 2nd International Semantic Web Conference-Workshop on Human Language Technology for the Semantic Web abd Web Services, Sanibel Island, FL, USA, 19-22 October 2003. 
17. Castano, S.; Peraldi, I.S.E.; Ferrara, A.; Karkaletsis, V.; Kaya, A.; Möller, R.; Montanelli, S.; Petasis, G.; Wessel, M. Multimedia Interpretation for Dynamic Ontology Evolution. J. Log. Comput. 2009, 19, 859-897. [CrossRef]

18. Suchanek, F.M. LEILA: Learning to Extract Information by Linguistic Analysis. In Proceedings of the 2nd Workshop on Ontology Learning and Population: Bridging the Gap between Text and Knowledge, Sydney, Australia, 22 July 2006; pp. 18-25.

19. Buitelaar, P.; Cimiano, P.; Racioppa, S.; Siegel, M. Ontology-based Information Extraction with SOBA. In Proceedings of the International Conference on Language Resources and Evaluation, Genoa, Italy, 22-28 May 2006; pp. 2321-2324.

20. Navigli, R.; Velardi, P. Enriching a Formal Ontology with a Thesaurus: An Application in the Cultural Heritage Domain. In Proceedings of the 2nd Workshop on Ontology Learning and Population, Bridging the Gap between Text and Knowledge, Sydney, Australia, 22 July 2006; pp. 1-9.

21. Sang, S.K.; Jeong-Woo, S.; Seong-Bae, P.; Lee, J.H.C.; Myung-Gil, J.; Hyung-Geun, P. PTIMA: A System for Semi Automatic and Large Scale Ontology Population. In Proceedings of the 3rd Workshop on Ontology Learning and Population, Patras, Greece, 21-22 July 2008; 2008.

22. Weber, N.; Buitelaar, P. Web-based Ontology Learning with ISOLDE. In Proceedings of the ISWC2006 Workshop on Web Content Mining with Human Language Technologies, Athens, GA, USA, 5-9 November 2006.

23. Brewster, C.; Ciravegna, F.; Wilks, Y. User-Centred Ontology Learning for Knowledge management. In Proceedings of the NLDB '02: 6th International Conference on Applications of Natural Language to Information Systems-Revised, Stockholm, Sweden, 27-28 June 2002; Springer: London, UK, 2002; pp. $203-207$.

24. Helmut, S. Probabilistic Part-of-Speech Tagging Using Decision Trees. In Proceedings of the International Conference on New Methods in Language Processing, Manchester, UK, 1994; Available online: http: //citeseerx.ist.psu.edu/viewdoc/summary?doi=10.1.1.28.1139 (accessed on 30 November 2020).

25. Giulio, G.; Domenico, L.; Massimo, M.; Federico, S. Ontology population for open-source intelligence: A GATE-based solution. J. Softw. Pract. Exp. 2018, 48, 2302-2330.

26. Ali, A.; Ahmed, S.; Francois, B.B.; Cecilia, Z.-M. Ontology population with deep learning based-NLP: A case study on the Biomolecular Network Ontology. In Proceedings of the 23rd International Conference on Knowledge-Based and Intelligent Information and Engineering Systems (Elsevier Procedia Computer Science), Budapest, Hungary, 23-24 July 2019; pp. 572-581.

27. Tomas, M.; Ilya, S.; Chen, K.; Corrado, G.; Jeffrey, D. Distributed representations of words and phrases and their compositionality. Adv. Neural Inf. Process. Syst. 2013, 26, 3111-3119.

28. Unbehauen, J.; Hellmann, S.; Auer, S.; Stadler, C. Knowledge Extraction from Structured Sources. In Search Computing_Broadening Web Search Lecture Notes in Computer Science; Springer: Berlin/Heidelberg, Germany, 2012; pp. 34-52.

29. Stevens, R.; Sattler, U. Disjointness between Classes in an Ontology. Ontogenesis 2012. Available online: http://ontogenesis.knowledgeblog.org/1260 (accessed on 20 September 2019).

30. Samir Tartir, I.; Arpinar, B.; Sheth, A.P. Ontological Evaluation and Validation. In Theory and Applications of Ontology: Computer Applications; Springer: New York, NY, USA, 2010; pp. 115-130.

31. Dittmann, L.; Rademacher, T.; Zelewski, S. Performing FMEA using ontologies. In Proceedings of the 18th International Workshop on Qualitative Reasoning, Evanston, IL, USA, 2-4 August 2004; pp. 209-216.

32. Ebrahimipour, V.; Rezaie, K.; Shokravi, S. An ontology approach to support FMEA studies. Expert Syst. Appl. 2010, 37, 671-677. [CrossRef]

33. Mikos, W.L.; Ferreira, J.C.; Botura, P.E.; Freitas, L.S. A system for distributed sharing and reuse of design and manufacturing. J. Manuf. Syst. 2011, 30, 133-143. [CrossRef]

Publisher's Note: MDPI stays neutral with regard to jurisdictional claims in published maps and institutional affiliations. 